\title{
An Improved Empirical Fuel Cell Polarization Curve Model Based on Review Analysis
}

\author{
Dong Hao, ${ }^{1,2}$ Jianping Shen, ${ }^{3}$ Yongping Hou, ${ }^{1,2}$ Yi Zhou, ${ }^{1,2}$ and Hong Wang ${ }^{4}$ \\ ${ }^{1}$ Lab of Clean Energy Automotive Engineering Center, Tongji University, Shanghai 201804, China \\ ${ }^{2}$ School of Automotive Studies, Tongji University, Shanghai 201804, China \\ ${ }^{3}$ Shanghai Motor Vehicle Inspection Center, Shanghai 201805, China \\ ${ }^{4}$ China National Institute of Standardization, Beijing 100088, China
}

Correspondence should be addressed to Yongping Hou; yphou@tongji.edu.cn

Received 20 November 2015; Revised 9 March 2016; Accepted 18 April 2016

Academic Editor: Iftekhar A. Karimi

Copyright (c) 2016 Dong Hao et al. This is an open access article distributed under the Creative Commons Attribution License, which permits unrestricted use, distribution, and reproduction in any medium, provided the original work is properly cited.

\begin{abstract}
Based on a review analysis of empirical fuel cell polarization curve models in the literature, an improved model that can predict fuel cell performance with only measured current-voltage data is developed. The fitting characteristics of this new model are validated by fitting bench test data and road test data. In the case of bench test data, a comparison of the new model and two representative models is conducted, and the results show that the new model presents the best fitting effects over a whole range of current densities. Moreover, the fitted ohmic resistances derived from the new model show good agreement with the measured values obtained through a current interruption test. In the case of using road test data, the new model also presents excellent fitting characteristics and convenience for application. It is the author's belief that the new model is beneficial for the application-oriented research of fuel cells due to its prominent features, such as conciseness, flexibility, and high accuracy.
\end{abstract}

\section{Introduction}

A polymer electrolyte membrane fuel cell (PEMFC) is an electrochemical device that converts chemical energy stored in hydrogen directly to electricity and heat with water as the only byproduct of the reaction. With the prominent features of zero emissions, low operating temperature, quick startup, and high efficiency, PEMFCs have been broadly considered the best electrical energy sources for automotive, stationary, and portable power devices [1]. In the area of PEMFCs research, mathematical fuel cell models, including mechanism and empirical models, are widely applied in the design, control, and optimization of PEMFCs [2-7]. Compared with mechanism models, empirical PEMFC polarization curve models are generally used in the application-oriented investigations and industrial research with advantages such as flexibility, simplicity, and acceptable accuracy.

For PEMFC, the polarization curve, which describes the relationship between output voltage and current density, is the most important characteristic of performance. Thus, a variety of empirical fuel cell polarization curve models have been developed in the past decades to reproduce the measured polarization curves containing a series of currentvoltage data points. Furthermore, the values of fitting parameters derived from the empirical models are also valuable references for the investigation of PEMFCs.

This work began with a review on existing empirical fuel cell polarization curve models. Based on this, a new empirical model for the entire range of current densities was then presented. Validation of the new model was executed through fitting bench test data and road test data. Regarding bench tests, a polarization curve test and current interruption test were carried out on a fuel cell stack consisting of 57 cells. Taking the measured current-voltage data as reference values, the fitting accuracy of the new model was investigated and compared with two representative models. Finally, the validity of the new model was discussed by comparing the fitted ohmic resistances with the experimental values. Regarding road tests, some discrete current-voltage data of a 90 -cell stack, which were sampled from a demonstrating fuel 
cell sightseeing vehicle, were used to analyze the application effects of different models.

\section{Review Analysis of Existing Polarization Curve Models}

With the aim of improving fitting accuracy of fuel cell performance throughout the range of operation, a number of researchers have developed numerous empirical and semiempirical fuel cell polarization curve models since the early 1990s. Ten polarization curve models are reviewed in this section. To distinguish fitting parameters from other parameters or constants, fitting parameters are written in boldface throughout this work.

The first empirical fuel cell polarization curve model with five fitting parameters was presented by Kim et al. [8] (denoted by model K):

$$
E_{\text {cell }}=\mathbf{E}_{\mathbf{O}}-\mathbf{b} \log (i)-\mathbf{R} i-\mathbf{m} \exp (\mathbf{n} i) .
$$

The parameter $\mathbf{E}_{\mathbf{O}}$ can be described as

$$
\mathbf{E}_{\mathbf{O}}=E_{\text {rev }}+\mathbf{b} \log \left(i_{o}\right),
$$

where $\mathbf{b} \log (i)$ represents activation loss, $\mathbf{R} i$ dedicates ohmic loss, and $\mathbf{m} \exp (\mathbf{n} i)$ is an empirical term that approximates mass transfer loss. It is noteworthy that $\mathbf{E}_{\mathbf{O}}$ is simply a fitting parameter rather than the open circuit voltage $(\mathrm{OCV})$ or the reversible voltage of the fuel cell. Fitting (1) has been cited by many studies and publications because of its advantages; that is, it fits the current-voltage data over the entire range of current densities under different temperatures, pressures, gas compositions, and so on. However, the measured value of OCV, the corresponding voltage of $0 \mathrm{~A} \mathrm{~cm}^{-2}$, cannot be used during the fitting process because of the $\log (i)$ term. In addition, although this equation provides excellent fitting characteristics with both medium and high current densities, there is a minor deficiency in fitting accuracy with small current densities.

Based on model K, several improved models aiming at modifying the term of mass transfer loss have been proposed. The model suggested by Lee et al. [9] is expressed as

$$
\begin{aligned}
E_{\text {cell }}= & \mathbf{E}_{\mathbf{O}}-\mathbf{b} \log (i)-\mathbf{R} i-\mathbf{m} \exp (\mathbf{n} i) \\
& -\mathbf{b} \log \left(\frac{p}{p_{\mathrm{O}_{2}}}\right),
\end{aligned}
$$

where the last term, containing the pressure ratio logarithm, is a form of the Nernst equation, which is introduced primarily to describe potential changes in the cathode. This term is an empirical constant parameter that has no relationship with current density. In addition, the pressure of oxygen must be measured before using this model.

The model of Squadrito et al. [10] is

$$
E_{\text {cell }}=\mathbf{E}_{\mathbf{O}}-\mathbf{b} \log (i)-\mathbf{R} i+\boldsymbol{\alpha}_{\mathbf{S}}{ }^{\mathbf{k}} \ln \left(1-\boldsymbol{\beta}_{\mathbf{S}} i\right),
$$

where $\boldsymbol{\alpha}_{\mathbf{S}} i^{\mathbf{k}}$ accounts for an "amplification term" of the logarithmic term; both $\boldsymbol{\alpha}_{\mathrm{S}}$ and $\mathbf{k}$ are fitting parameters without any physical meaning; $\boldsymbol{\beta}$ is a fitting parameter representing the inverse of the limiting current density. This model improves the fitting characteristic of the mass transfer loss term by increasing a fitting parameter.

The model of Chu et al. [11] can be described as

$$
\begin{aligned}
E_{\text {cell }} & =\mathbf{E}_{\mathbf{O}}-\mathbf{b} \log (i)-\mathbf{R} i-i_{m} \mathbf{m} \exp \left(\mathbf{n} i_{m}\right), \\
i_{m} & =i-i_{d} \quad\left(\text { for } i>i_{d}\right), \\
i_{m} & =0 \quad\left(\text { for } i \leq i_{d}\right),
\end{aligned}
$$

where $i_{d}$ is the smallest current density that causes the voltage to deviate from linearity. When the current density is less than $i_{d}$, the term of mass transfer loss in (5) is equal to 0 . That is, mass transfer loss occurs only in the high current density region; nevertheless, in fact, it occurs over the entire range of current densities. With the foregoing considerations, an improved model was proposed by Xia and Chan [12]:

$$
\begin{aligned}
E_{\text {cell }} & =\mathbf{E}_{\mathbf{O}}-\mathbf{b} \log (i)-\mathbf{R} i-i \mathbf{m} \exp \left(\mathbf{n} i_{m}\right), \\
i_{m} & =i-i_{d} \quad\left(\text { for } i>i_{d}\right), \\
i_{m} & =0 \quad\left(\text { for } i \leq i_{d}\right) .
\end{aligned}
$$

In this model, first $i_{m}$ in (5) is substituted by parameter $i$. As a result, when the current density is less than $i_{d}$, the mass transfer loss in (8) is equal to $i \mathbf{m}$, which means that the relationship between mass transfer loss and current density is linear in the low and moderate current density range. The coefficient $\mathbf{m}$ indicates that the mass transfer phenomenon occurs over the entire range of current densities. The nonlinear $\exp \left(\mathbf{n} i_{m}\right)$ term indicates that the phenomenon occurs at current densities that are higher than $i_{d}$.

Similarly, some modifications on the mass transfer loss term were executed by Pisani et al. [13]:

$$
E_{\text {cell }}=\mathbf{E}_{\mathbf{O}}-\mathbf{b} \log (i)-\mathbf{R} i+\mathbf{a}_{\mathbf{P}} \ln \left(1-\frac{i}{\mathbf{i}_{\mathbf{l}}} \mathbf{S}^{-\mu\left(1-i / \mathbf{i}_{\mathbf{1}}\right)}\right),
$$

where $\mathbf{S}$ is a fitting parameter describing the flooding phenomenon and $\mu$ is an empirical constant. The derivation of this model is based on the observation that the strongest nonlinear contributions to the cell voltage drop at high current densities arise from interface phenomenon happening in the cathode reactive region. One of the limitations of this model is the fact that there are three fitting parameters and one empirical constant to be included in the mass transfer loss term.

In summary, (3)-(5), (8), and (11) are obtained by modifying the term of mass transfer loss in model $\mathrm{K}$. Each of these models consists of a constant voltage fitting parameter, a logarithmic term approximating the activation loss, a linear term representing the ohmic loss, and one or two terms describing the mass transfer loss. As with model $\mathrm{K}$, the parameters of $\mathbf{E}_{\mathbf{O}}$ in these models are merely fitting parameters without any theoretical meanings. Moreover, owing to the existence of the term $\log (i)$, which tends towards infinity when the current density decreases to zero, it is difficult to precisely predict the OCV and the performance at small current densities. 
Considering that the models mentioned above cannot accurately fit no-load operation and small current densities, Fraser and Hacker [14] executed some modifications to (1) and presented a model (denoted by model F) as follows:

$$
E_{\mathrm{cell}}=E_{\mathrm{rev}}-\mathbf{b} \log \left(\frac{i+\mathbf{i}_{\text {loss }}}{\mathbf{i}_{\mathbf{o}}}\right)-\mathbf{R} i-\mathbf{m} \exp (\mathbf{n} i) .
$$

The reversible voltage, which is a constant term, can be estimated with known fuel cell operation temperature and partial pressures of oxygen and hydrogen. Additional fitting parameters $\mathbf{i}_{\text {loss }}$ and $\mathbf{i}_{\mathbf{o}}$ are introduced into the logarithmic term. Consequently, this equation provides accurate fitting characteristics with small current densities and OCV. It is worth noting that the reversible voltage here is merely an approximate value because it is difficult to measure the actual values of the operation temperature and partial pressures of the reactants.

In the case of a hydrogen/air fuel cell, the thermodynamic reversible voltage, $E_{\text {rev }}$, is calculated from the modified Nernst equation, which considers both temperature and pressure changes [9]:

$$
E_{\mathrm{rev}}=E^{0}+\frac{\Delta \widehat{s}}{n_{e} F}\left(T-T_{0}\right)-\frac{R_{g} T}{n F} \ln \left(\frac{a_{\mathrm{H}_{2} \mathrm{O}}}{a_{\mathrm{H}_{2}} a_{\mathrm{O}_{2}}^{1 / 2}}\right),
$$

where $E^{0}$ is the standard-state reversible voltage $(1.229 \mathrm{~V})$ and $\Delta \widehat{s}$ is the entropy of the reaction (assuming that it is independent of temperature), which is $-163.28 \mathrm{~J} \mathrm{~mol}^{-1} \mathrm{~K}^{-1}$ at temperature $T_{0} \cdot a_{x}$ is the activity of $x$. For an ideal gas, $a_{x}=p_{x} / p^{0}$, where $p_{x}$ is the partial pressure of gas $x$ and $p^{0}$ is the standard-state pressure (1 atm). For a hydrogen/air fuel cell operating at a low temperature with liquid water as a product, the activity of water is 1 .

By substituting the known parameters mentioned above into (13), the reversible voltage can be described as a function of temperature and pressure as follows:

$$
\begin{aligned}
E_{\mathrm{rev}}= & 1.229-8.46 \times 10^{-4}(T-298.15)-4.31 \\
& \times 10^{-5} T \ln \left(\frac{1}{p_{\mathrm{H}_{2}} p_{\mathrm{O}_{2}}^{1 / 2}}\right)
\end{aligned}
$$

As shown by (14), the operation temperature of the fuel cell and partial pressures of oxygen and hydrogen are indispensable for calculating the value of the reversible voltage. To reduce the number of indispensable measured parameters in the model, a fitting term of reversible voltage was used in (15) by Weydahl et al. [15] and in (16) by Poh et al. [16]:

$$
\begin{aligned}
E_{\mathrm{cell}}= & \mathbf{E}_{\mathrm{rev}}-\mathbf{b} \log \left(\frac{i+\mathbf{i}_{\text {loss }}}{\mathbf{i}_{\mathbf{o}}}\right)-\mathbf{R}\left(i+\mathbf{i}_{\text {loss }}\right) \\
& +\mathbf{c} \ln \left(1-\frac{i+\mathbf{i}_{\text {loss }}}{\mathbf{i}_{\text {lim }}}\right), \\
E_{\text {cell }}= & \mathbf{E}_{\mathrm{rev}}-\mathbf{b} \log \left(\frac{i+\mathbf{i}_{\text {loss }}}{\mathbf{i}_{\mathbf{o}}}\right)-\mathbf{R} i-i \mathbf{m} \exp (\mathbf{n} i) .
\end{aligned}
$$

Either (15) or (16) contains the maximum number of fitting parameters among these ten existing models. Compared with model $\mathrm{K}$, two additional fitting parameters are introduced. To regard the reversible voltage as a fitting parameter, it is difficult to obtain a unique, reasonable solution of $\mathbf{E}_{\mathrm{rev}}$. As a consequence, there are many solutions for other fitting parameters.

Equation (17), developed by Haji [17], also regards the reversible voltage as a constant term and presents outstanding fitting accuracy over the entire region of current densities. Consider

$$
\begin{aligned}
E_{\text {cell }}= & E_{\text {rev }}-\left[\mathbf{a}+\mathbf{b} \ln \left(i+i_{\text {loss }}\right)\right]-\mathbf{R} i \\
& -\mathbf{c} \ln \left(\frac{i_{\text {lim }}}{i_{\text {lim }}-\left(i+i_{\text {loss }}\right)}\right) .
\end{aligned}
$$

Although there are only four fitting parameters in this model, the limiting current density and current loss are obtained through measurements. As a result, the range of application of this model is limited.

In addition, an analysis technique to evaluate six sources of polarization losses in hydrogen/air proton exchange membrane fuel cells was developed by Williams et al. [18]. The overall polarization curve was described by two separate equations: one for current densities smaller than $i_{b}$ and one for current densities greater than $i_{b}$. At

$$
\begin{aligned}
i<i_{b} & \\
E_{\text {cell }}= & E_{\text {rev }}+b \log \left(i_{\mathbf{o}}\right)-b \log (i)-i R_{\text {electrode }} \\
& -i R_{\text {nonelectrode }}
\end{aligned}
$$

At

$$
\begin{aligned}
i \geq & i_{b} \\
E_{\text {cell }}= & E_{\text {rev }}+b^{\prime} \log \left(i_{\mathbf{o}}^{\prime}\right)-b^{\prime} \log \left[\frac{i}{\left(1-i / i_{\text {lim }}\right)}\right] \\
& \quad-i R_{\text {electrode }}-i R_{\text {nonelectrode }}
\end{aligned}
$$

The approach in their work is very clear and effective for evaluating different sources of polarization losses under laboratory conditions. Based on determinations of some parameters, including reversible voltage, nonelectrode ohmic resistance, cathode electrode ohmic resistance, limited current density, Tafel slope, and exchange current density, the output voltage of fuel cells is calculated. The development of this model is aimed at distinguishing different polarization losses in fuel cells rather than reproducing the whole polarization curve based on voltage/current data. However, it provided useful insights for analyzing the polarization curve to future works on developing a model without any parameters that lack physical significance.

In conclusion, all of the models reviewed above have their merits and drawbacks, and their numbers of fitting parameters range from 4 to 7 . The shortages and inconveniences of these models are listed in Table 1.

After reviewing existing models of polarization curves, it can be concluded that model K [8] and model F [14] are the 
TABLE 1: Shortages and inconveniences of existing empirical fuel cell polarization curve models.

\begin{tabular}{|c|c|c|c|c|c|c|c|c|c|c|}
\hline \multirow{2}{*}{ Features } & \multicolumn{10}{|c|}{ Equation number } \\
\hline & (1) & (3) & (4) & (5) & $(8)$ & (11) & $(12)$ & (15) & (16) & (17) \\
\hline Number of fitting parameters & 5 & 5 & 6 & 5 & 5 & 6 & 6 & 7 & 7 & 4 \\
\hline Unavailable to fit OCV & $\sqrt{ }$ & $\sqrt{ }$ & $\sqrt{ }$ & $\sqrt{ }$ & $\sqrt{ }$ & $\sqrt{ }$ & & & & \\
\hline Low fitting accuracy of small current densities & $\sqrt{ }$ & $\sqrt{ }$ & $\sqrt{ }$ & $\sqrt{ }$ & $\sqrt{ }$ & $\sqrt{ }$ & & & & \\
\hline Requiring estimating $E_{\text {rev }}$ or low fitting accuracy of $\mathbf{E}_{\text {rev }}$ & & & & & & & $\sqrt{ }$ & $\sqrt{ }$ & $\sqrt{ }$ & $\sqrt{ }$ \\
\hline Requiring measured parameters, such as temperature and gas partial pressure & & $\sqrt{ }$ & & & & & $\sqrt{ }$ & & & $\sqrt{ }$ \\
\hline Requiring estimating $i_{d}$ & & & & $\sqrt{ }$ & $\sqrt{ }$ & & & & & \\
\hline Mass transfer loss occurring only in high current density region & & & & $\sqrt{ }$ & & & & & & \\
\hline Requiring other empirical constants & & & & & & $\sqrt{ }$ & & & & \\
\hline Requiring measured $i_{\text {lim }}$ and $i_{\text {loss }}$ & & & & & & & & & & $\sqrt{ }$ \\
\hline
\end{tabular}

two most representative models. Hence, these two models are chosen as the references for comparison with the new model given in the following part of this work.

\section{Improved Empirical Polarization Curve Model}

In this work, a series of modifications is performed on the simplified version of theoretical fuel cell polarization curve models, and an improved empirical model is then obtained. The processes of modifications are elaborated as follows.

The fuel cell output voltage can be expressed by the following equation:

$$
E_{\text {cell }}=E_{\text {rev }}-\Delta V_{\text {act }}-\Delta V_{\text {ohmic }}-\Delta V_{\text {conc }}
$$

The activation loss, $\Delta V_{\text {act }}$, is described by the ButlerVolmer equation and is associated with sluggish electrode kinetics. The Butler-Volmer equation can be reduced to the Tafel equation:

$$
\Delta V_{\mathrm{act}}=a+b \log (i)
$$

where

$$
\begin{aligned}
& a=-2.3 \frac{R_{g} T}{\alpha F} \log \left(i_{o}\right) \\
& b=2.3 \frac{R_{g} T}{\alpha F} .
\end{aligned}
$$

Term $b$ is called the Tafel slope. Hence, the activation loss can be given in a simplified form:

$$
\Delta V_{\text {act }}=b \log \left(\frac{i}{i_{o}}\right) .
$$

The ohmic loss, $\Delta V_{\text {ohmic }}$, which obeys Ohm's law, occurs because of resistance to the flow of ions in the electrolyte and resistance to the flow of electrons through the electrically conductive fuel cell components. Consider

$$
\Delta V_{\text {ohmic }}=i R \text {. }
$$

The mass transfer loss, $\Delta V_{\text {conc }}$, occurs when reactants are rapidly consumed at the electrode by the electrochemical reaction so that concentration gradients are established. The voltage loss caused by the mass transport limitation is expressed by the following equation:

$$
\Delta V_{\text {conc }}=\frac{R_{g} T}{n_{e} F} \ln \left(\frac{i_{\text {lim }}}{i_{\text {lim }}-i}\right) .
$$

In addition to the activation, ohmic and mass transfer losses, current losses due to hydrogen permeation, and electron crossover also exist in fuel cells. Neglecting the effect of current losses on ohmic loss and mass transfer loss, a simplified version of the theoretical fuel cell polarization curve model that consists of numerous parameters can be described as [19]

$$
\begin{aligned}
E_{\text {cell }}= & E_{\text {rev }}-b \log \left(\frac{i+i_{\text {loss }}}{i_{o}}\right)-R i \\
& -\frac{R_{g} T}{n_{e} F} \ln \left(\frac{i_{\text {lim }}}{i_{\text {lim }}-i}\right) .
\end{aligned}
$$

When external current density $i$ equals zero, the fuel cell operates as an open circuit, so (26) can be expressed as

$$
E_{\mathrm{OCV}}=E_{\mathrm{rev}}-b \log \left(\frac{i_{\text {loss }}}{i_{o}}\right) .
$$

The following equation can then result from (27):

$$
E_{\mathrm{rev}}=E_{\mathrm{OCV}}+b \log \left(\frac{i_{\text {loss }}}{i_{o}}\right) .
$$

In a fuel cell, as the cell current becomes high, which indicates that the electrochemical reaction rate on the electrode surface is fast, the mass transfer rate of the reactants is not sufficiently fast to provide enough reactants to the electrode surface. Depletion of reactants at the electrode surface leads to a drop in cell voltage. The calculation of the cell voltage drop in this part is difficult in some conditions without all operational parameters, so the empirical equation suggested by Kim et al. [8] is usually used to estimate the mass transfer drop:

$$
\Delta V_{\text {conc }}=\mathbf{m} \exp (\mathbf{n} i) .
$$


The term of mass transfer loss, $\mathbf{m} \exp (\mathbf{n} i)$, is proposed based on experimental data with two zero physical significant parameters. However, for practical purposes, the adoption of these parameters is meaningful.

When a fuel cell is under no-load operation, the value of (29) is $\mathbf{m}$, which does not conform to zero for the theoretical mass transfer loss. Hence, to set the empirical term of mass transfer loss equal to zero under the open circuit condition, (29) is modified into the following form:

$$
\Delta V_{\text {conc }}=\mathbf{m}[\exp (\mathbf{n} i)-1] .
$$

Combined with (26), (28), and (30), a new empirical fuel cell polarization curve model can be written as

$$
\begin{aligned}
E_{\text {cell }}= & E_{\mathrm{OCV}}-\mathbf{b} \log \left(\frac{i+\mathbf{i}_{\text {loss }}}{\mathbf{i}_{\text {loss }}}\right)-\mathbf{R} i \\
& -\mathbf{m}[\exp (\mathbf{n} i)-1]
\end{aligned}
$$

where $E_{\mathrm{OCV}}$ is known as a measured value; five fitting parameters, $\mathbf{b}, \mathbf{i}_{\text {loss }}, \mathbf{R}, \mathbf{m}$, and $\mathbf{n}$, are adopted.

Neglecting the mass transfer loss, the new model can be expressed as (32). The neglect of the mass transport limitation results in a simple model that is generally used to represent the low and moderate current density regions of a fuel cell polarization curve in which only slight mass transfer loss appears. Consider

$$
E_{\text {cell }}=E_{\mathrm{OCV}}-\mathbf{b} \log \left(\frac{i+\mathbf{i}_{\text {loss }}}{\mathbf{i}_{\text {loss }}}\right)-\mathbf{R} i .
$$

In this simplified formation, there are three fitting parameters: $\mathbf{b}, \mathbf{i}_{\text {loss }}$, and $\mathbf{R}$.

The new model (denoted model N) can be applied to represent measured polarization curves with only currentvoltage data. Meanwhile, the application of this model does not require reversible voltage and complicated operational parameters, such as the fuel cell temperature and partial pressures of reactants. As a result of the modifications of the logarithmic term and mass transfer loss term, the new model is available to accurately fit very small current densities, and the fitted polarization curve passes through the test OCV absolutely.

The assumptions, approximations, and limitations of the proposed model are illustrated as follows:

(i) Assume that the anode losses of fuel cells are negligible compared with the cathode losses.

(ii) Neglect the effect of current losses on ohmic loss and mass transfer loss.

(iii) The activation polarization is described by the Tafel equation.

(iv) The ohmic loss is expressed by Ohm's law, and the ohmic resistance remains constant over the whole range of current density.

(v) Assume that the mass transfer loss approximates $\mathbf{m}[\exp (\mathbf{n} i)-1]$.
TABLE 2: Operational conditions of the fuel cell stack (bench test).

\begin{tabular}{lc}
\hline Parameters & Values \\
\hline Relative humidity of hydrogen & Without humidification \\
Relative humidity of air & $80 \%$ \\
Stoichiometry of hydrogen & 1.2 \\
Stoichiometry of air & 2.5 \\
Hydrogen inlet temperature & $60^{\circ} \mathrm{C}$ \\
Air inlet temperature & $60^{\circ} \mathrm{C}$ \\
Hydrogen inlet pressure & 1.51 atm \\
Air inlet pressure & Without pressurization \\
Coolant outlet temperature & Approximately $60^{\circ} \mathrm{C}$ \\
\hline
\end{tabular}

(vi) The model can be used under all conditions (including different operational conditions and component materials) as long as the voltage-current data of polarization curves are measured.

\section{Results and Discussion}

4.1. Validation Using Bench Test Data. The comparison of the measured and fitted fuel cell performance is carried out with a polarization curve derived from a fresh commercial $8 \mathrm{~kW}$ fuel cell stack. The stack consists of 57 cells, which are assembled in series. The active area per single cell is $312 \mathrm{~cm}^{2}$. The fuel cell stack test station for this study is G500, designed by Greenlight Innovation, Canada [20]. The G500 is a complete fuel cell testing solution that includes precise gas flow control using mass flow controllers, accurate gas humidification, stack coolant, proprietary pressure control, expandable cell voltage monitoring capability, and a watercooled load bank with a maximum power of $30 \mathrm{~kW}$. The operational conditions of the fuel cell stack are shown in Table 2.

The polarization curve test of fuel cell stack was conducted under a constant gas stoichiometry condition. The current-voltage characteristics were obtained by varying the current density from 0 to the maximum value at a suitable interval and maintaining each current density until the average voltage of a single cell was stabilized within $\pm 5 \mathrm{mV}$ for $3 \mathrm{~min}$ [21]. The measured polarization curve of a fuel cell stack that has been normalized to the average of a single cell is shown by the black line in Figure 1. The measured OCV of the fuel cell stack is $0.9560 \mathrm{~V}$, which plays a significant role in model N.

In addition, the ohmic resistances of the fuel cell stack are obtained through a current interruption test. The average ohmic resistances of single cells, corresponding to different current densities, are acquired by dividing the ohmic resistances of the fuel cell stack by the cell number of 57 and the range from $0.1062 \Omega \mathrm{cm}^{2}$ to $0.1086 \Omega \mathrm{cm}^{2}$ with an average value of $0.1071 \Omega \mathrm{cm}^{2}$, as depicted in Figure 1 .

4.1.1. Validation of New Model. To validate the new model, the fitting characteristics are investigated and compared with those of model $\mathrm{K}$ and model $\mathrm{F}$. Model $\mathrm{N}$ and model $\mathrm{K}$ can fit the test data directly, whereas model $\mathrm{F}$ requires the value 


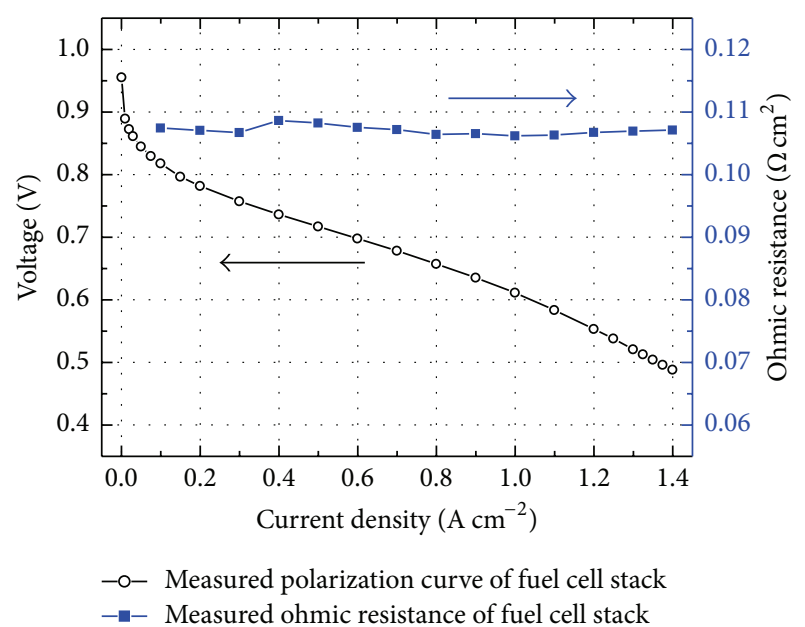

FIGURE 1: Measured polarization curve and ohmic resistances of the fuel cell stack.

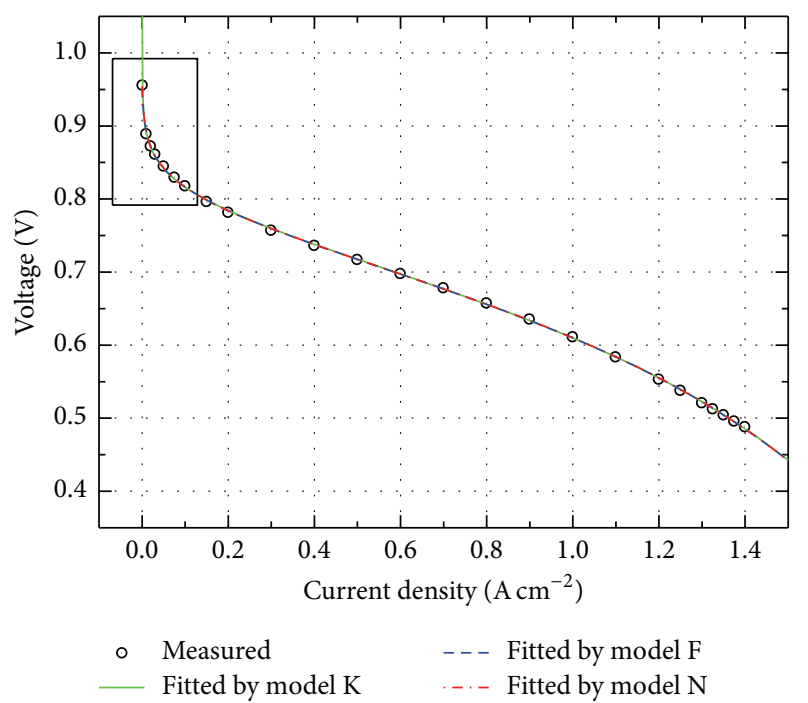

Figure 2: Comparison of measured and fitted polarization curves (bench test).

of reversible voltage. As shown in Table 2, the hydrogen and oxygen partial pressures are $1.51 \mathrm{~atm}$ and $0.21 \mathrm{~atm}(1 \times$ $21 \% \mathrm{~atm})$, respectively. The operational temperature of the fuel cell stack is approximately $333.15 \mathrm{~K}$. Consequently, a reversible voltage of $1.1940 \mathrm{~V}$ is obtained by substituting the abovementioned values into (14).

The three fitted polarization curves and measured current-voltage data are depicted in Figure 2. It is clear that these three models exert excellent fitting characteristics with both medium and high current densities. However, whereas the current density declines to zero, the voltage fitted by model $\mathrm{K}$ increases dramatically and tends towards infinity. The region of small current densities in Figure 2 is presented in Figure 3. It can be observed that the curve fitted by model $\mathrm{K}$ deviates from the other two fitting curves significantly in a very small current density region (approximately 0 $0.01 \mathrm{~A} / \mathrm{cm}^{2}$ ).

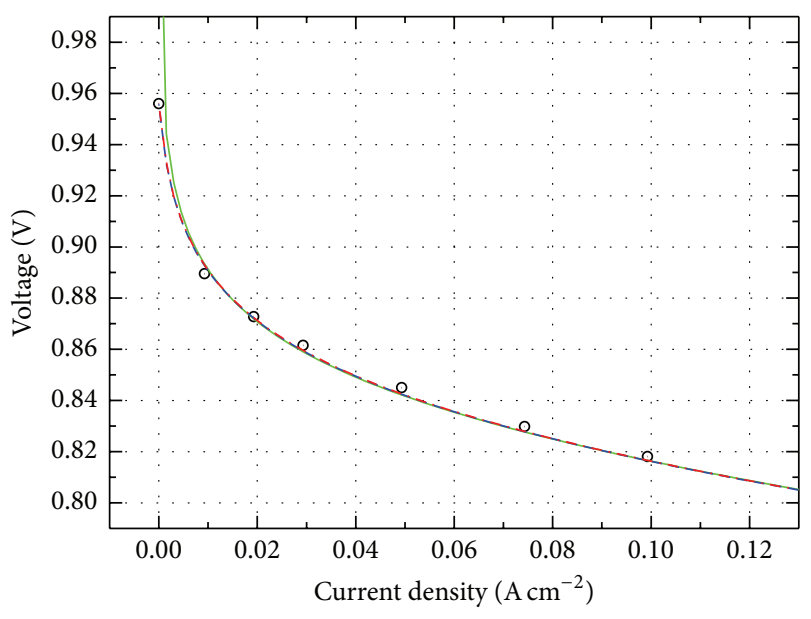

- Measured --- Fitted by model F

_ Fitted by model K _..- Fitted by model $\mathrm{N}$

FIGURE 3: Comparison of measured and fitted polarization curves (small current densities) (bench test).

To evaluate the prediction accuracy of these three models in terms of quantity over the whole region of current densities, the fitted voltages of each model are compared with the measured values at different current densities (25 test points in total). For model $\mathrm{K}$, because the fitted voltage corresponding to $0 \mathrm{~A} / \mathrm{cm}^{2}$ cannot be obtained, the fitted voltage at $1 \mathrm{~mA} \mathrm{~cm}^{-2}$ is used here as a substitution of OCV.

The absolute deviation between fitted voltage and measured voltage is defined as

$$
d_{\text {fit }}\left(i_{p}\right)=\left|E_{\text {fit }}\left(i_{p}\right)-E_{\text {test }}\left(i_{p}\right)\right|,
$$

where $i_{p}$ is the current density of test point $p$ and $E_{\mathrm{fit}}\left(i_{p}\right)$ and $E_{\text {test }}\left(i_{p}\right)$ are the fitted voltage and measured voltage at $i_{p}$, respectively. All absolute deviations of the three models are less than $5 \mathrm{mV}$, which meets the requirement for voltage stabilization in the polarization curve test, as depicted in Figure 4. It is obvious that only the OCV fitted by model N has no deviation from the measured value. That is, compared with model $\mathrm{K}$ and model $\mathrm{F}$, the polarization curve fitted by model $\mathrm{N}$ completely passes through the measured OCV. For the region of $0-0.5 \mathrm{~A} \mathrm{~cm}^{-2}$, the fitting accuracy of model $\mathrm{K}$ is slightly lower than those of the other two models. For the region of $0.5-1.4 \mathrm{~A} \mathrm{~cm}^{-2}$, the three models present almost the same fitting accuracy.

The average of the absolute deviations between fitted voltage and measured voltage is defined as (34), and the results are given in Table 3. Consider

$$
\overline{d_{\text {fit }}}=\frac{\sum_{p=1}^{25} d_{\text {fit }}\left(i_{p}\right)}{25} .
$$

As illustrated in Table 3, the average of the absolute deviations derived from model $\mathrm{N}$ is the minimum among the three values. As a result, model $\mathrm{N}$ exhibits the best fitting accuracy in the entire region of current densities. Compared with model F, data that are difficult to measure and estimate 
TABLE 3: Average and maximum values of absolute deviations derived from three models.

\begin{tabular}{lccc}
\hline & Model K & Model F & Model N \\
\hline Average of absolute deviations $(\mathrm{mV})$ & 1.5919 & 1.4883 & 1.4642 \\
Maximum absolute deviation $(\mathrm{mV})$ & 3.8224 & 3.1011 & 3.4242 \\
\hline
\end{tabular}

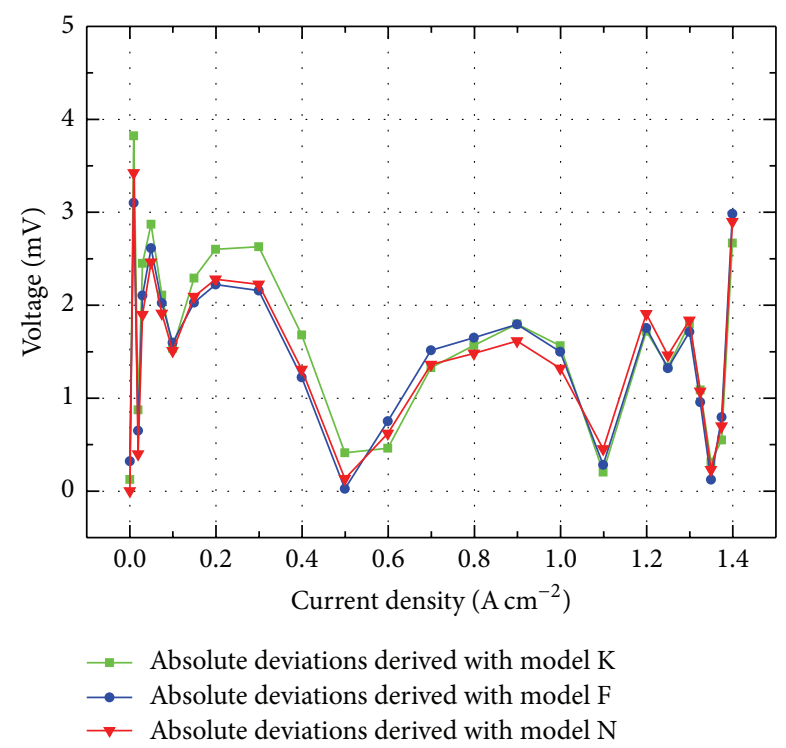

FIGURE 4: Absolute deviations of fitted voltage and measured voltage derived from the three models.

are nonessential to the application of model N. In addition, the fitting parameter for exchange current density in model $\mathrm{F}$ is eliminated so that the structure of model $\mathrm{N}$ is much more compact.

The values of fitting parameters obtained from the three models are listed in Table 4. As seen in the table, the fitted values of $\mathbf{b}, \mathbf{R}, \mathbf{m}$, and $\mathbf{n}$ derived from each of the three models exhibit slight differences. The value of $\mathbf{E}_{\mathbf{O}}$ derived from model $\mathrm{K}$ is unreasonable and much smaller than the other two values. The application of the $\log (i)$ term in model $\mathrm{K}$ is the main reason for this result. Model $\mathrm{F}$ predicts the OCV approximately, whereas model $\mathrm{N}$ predicts the OCV perfectly. Regarding the ohmic resistance $\mathbf{R}$ and Tafel slope $\mathbf{b}$, the results obtained from model $\mathrm{N}$ approximate those of model $\mathrm{F}$ and obviously deviate from those of model K. A slight distinction is detected between the internal current densities derived with model $\mathrm{F}$ and model $\mathrm{N}$, and both of them are reasonable [19].

4.1.2. Analysis of Fitted Ohmic Resistance. During the polarization curve test, current-voltage data of every single cell in the stack have also been acquired. The polarization curves of 57 single cells are fitted by model $\mathrm{N}$ such that the values of fitting parameters can be investigated further.

The ohmic resistances of 57 single cells derived from model $\mathrm{N}$ are depicted in Figure 5. The bars represent the ohmic resistances fitted from 57 single cell polarization curves, and the dashed line shows their average with a
TABLE 4: Values of fitting parameters obtained from the three models (bench test).

\begin{tabular}{lccc}
\hline & Model K & Model F & Model N \\
\hline$E_{\text {rev }}(\mathrm{V})$ & N/A & 1.1940 & N/A \\
$E_{\text {OCV }}(\mathrm{V})$ & $0.7703\left(\mathbf{E}_{\mathbf{O}}\right)$ & 0.9557 & 0.9560 \\
$\mathbf{b}(\mathrm{V} /$ decade $)$ & $0.9559\left(\right.$ at $\left.1 \mathrm{~mA} \mathrm{~cm}^{-2}\right)$ & & \\
$\mathbf{R}\left(\Omega \mathrm{cm}^{2}\right)$ & 0.06362 & 0.06642 & 0.06677 \\
$\mathbf{i}_{\text {loss }}\left(\mathrm{A} \mathrm{cm}^{2}\right)$ & 0.1121 & 0.1083 & 0.1073 \\
$\mathbf{i}_{\mathbf{o}}\left(10^{-7} \mathrm{~A} \mathrm{~cm}^{2}\right)$ & $\mathrm{N} / \mathrm{A}$ & 0.001225 & 0.001241 \\
$\mathbf{m}(\mathrm{V})$ & $\mathrm{N} / \mathrm{A}$ & 3.7980 & $\mathrm{~N} / \mathrm{A}$ \\
$\mathbf{n}\left(\mathrm{cm}^{2} \mathrm{~A}^{-1}\right)$ & 0.005165 & 0.005248 & 0.005339 \\
\hline
\end{tabular}

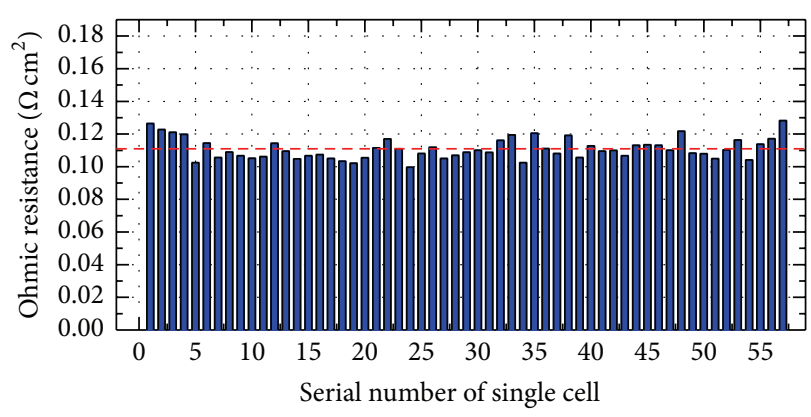

Fitted ohmic resistances of numbers $1-57$ cells Average of fitted ohmic resistances of numbers 1-57 cells

FIGURE 5: Fitted ohmic resistances of 57 single cells.

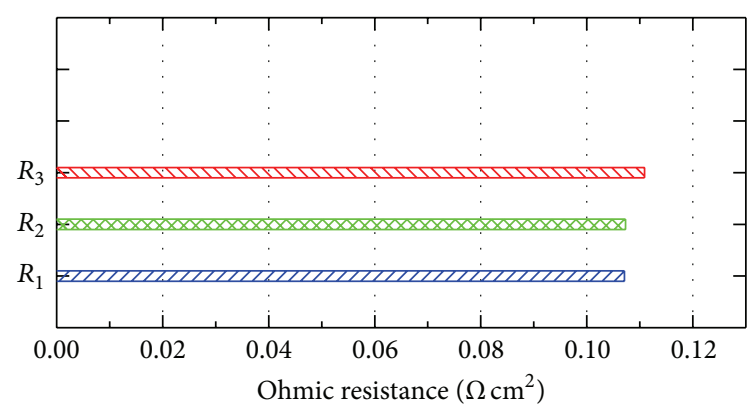

MV Average of fitted ohmic resistances of numbers 1-57 cells Fitted value of average ohmic resistance of single cell

VZA Reference value of average ohmic resistance of single cell

FIGURE 6: Comparison of ohmic resistances obtained from measurement and fits.

value of $0.1109 \Omega \mathrm{cm}^{2}$. The fitted ohmic resistances of 57 cells remain in a reasonable range [19] with a maximum value of $0.1282 \Omega \mathrm{cm}^{2}$ (cell number 57) and a minimum value of $0.0996 \Omega \mathrm{cm}^{2}$ (cell number 24).

Because the ohmic resistance of the fuel cell stack has been obtained through the current interruption test, the average value of the measured results, that is, $0.1071 \Omega \mathrm{cm}^{2}$, is taken as the reference value to the fitted values in what follows. As seen in Figure 6, $R_{1}$ and $R_{2}$ represent the reference and fitted values of the average ohmic resistance of a single 
cell, respectively. $R_{3}$ represents the average of the fitted ohmic resistances of cells numbers $1-57$, which corresponds to the dashed line in Figure 5. The fitted average ohmic resistance of a single cell is $0.1073 \Omega \mathrm{cm}^{2}$, larger than the reference value by $0.19 \%$. The average of 57 fitted ohmic resistances is $0.1109 \Omega \mathrm{cm}^{2}$, which is $3.55 \%$ larger than the reference value.

From the foregoing analysis, in addition to the excellent fitting characteristics of current-voltage data, the fitting results of the ohmic resistance derived with model $\mathrm{N}$ are in good agreement with the measured values and can be a reference for research on polarization curves.

The model in this work is developed based on a review of other existing models. The fitting accuracy of similar empirical models under different operational conditions and component materials has been corroborated by many other works, such as $[8,10-13,16,17]$. To be different from the previous works, the resistances were measured through a current interruption test, and the residuals of the voltage at different current densities were calculated. Because of the forgiving nature of the model, it is difficult to obtain a unique solution of a set of test data. The adoption of the least square method during the fitting process can ensure an optimal solution of parameters in an empirical model. Inevitably, the fitting values cannot be completely equal to the theoretical value, but they can be used as references to understand the voltage losses in fuel cells. Although some parameters in the proposed model cannot be measured, a comparison of fitting results between our results and those of other works was conducted. As shown in Table 4, the fitting values of parameters are similar to those obtained from two representative models. This corroboration method was also adopted in $[12,14]$.

4.2. Validation Using Road Test Data. To investigate the performance and durability of fuel cell stacks for automotive applications, road tests or demonstrations of fuel cell vehicles are significant and indispensable. Under a real road environment, a vehicular fuel cell stack experiences much more complicated and harsher operational conditions than those under bench tests, such as dynamic cycling and changes of ambient temperature, pressure, and relative humidity. Because of the frequent load change, the performance data of the fuel cell stack sampled from the road test are massive and discrete, in total contrast to those obtained from the bench test. As a consequence, the empirical fuel cell polarization curve model is an effective tool to extract the whole polarization curve from the complex data.

In this work, some experimental data, which are obtained from a demonstration fuel cell sightseeing vehicle with a sampling frequency of $1 \mathrm{~Hz}$, are used to compare the application effects of the three models, that is, model K, model F, and model $\mathrm{N}$. The fuel cell stack adopted in this sightseeing vehicle has a designed output power of $5.8 \mathrm{~kW}$ and consists of 90 cells assembled in series. The electrochemical active area per cell is $250 \mathrm{~cm}^{2}$. Under a real road environment, the known operational conditions of the stack are listed in Table 5.

Similarly, model $\mathrm{N}$ and model $\mathrm{K}$ can fit the discrete data directly, whereas model $\mathrm{F}$ requires the value of reversible voltage. According to the information in Table 5, hydrogen and oxygen partial pressures are approximately $1.6 \mathrm{~atm}$ and
TABLE 5: Operational conditions of the fuel cell stack (road test).

\begin{tabular}{lc}
\hline Parameters & Values \\
\hline Hydrogen inlet pressure & Approximately $1.6 \mathrm{~atm}$ \\
Air inlet pressure & Approximately $1.48 \mathrm{~atm}$ \\
Coolant outlet temperature & Approximately $55^{\circ} \mathrm{C}$ \\
\hline
\end{tabular}

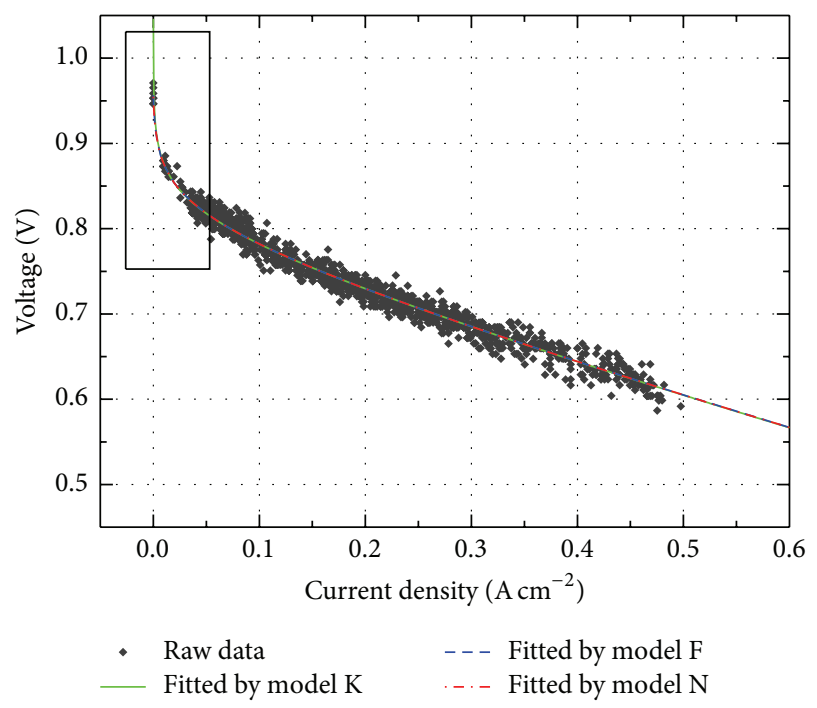

FIGURE 7: Comparison of measured and fitted polarization curves (road test).

0.3108 atm $(1.48 \times 21 \%$ atm $)$, respectively. The operational temperature of the stack is approximately $328.15 \mathrm{~K}$. Thus, an estimated reversible voltage of $1.2020 \mathrm{~V}$ is gained by substituting the known data into (14). Compared with bench test, it is much more difficult to accurately maintain steady operational conditions of the fuel cell stack during the road test owing to the complicated operation of the vehicle. As a consequence, it is difficult to obtain an exact value of the reversible voltage.

The raw data depicted in Figure 7 show that all output current densities of the fuel cell stack are less than $0.5 \mathrm{~A} \mathrm{~cm}^{-2}$. In addition, the shape of the scatterplot also does not present an apparent characteristic of the mass transport limitation. As a result, simple formations of three models similar to (32), which neglect the mass transfer loss terms containing $\mathbf{m}$ and $\mathbf{n}$, are adopted to fit the scattered data $[8,14]$. Another point to consider is that the value of $E_{\mathrm{OCV}}$ in model $\mathrm{N}$ is the average of sampled OCV in raw data, that is, $0.9576 \mathrm{~V}$.

As plotted in Figure 7, these three models present almost the same fitting results in the whole range of current densities, whereas the voltage fitted by model $\mathrm{K}$ increases dramatically and tends towards infinity when the current density approximates zero. As shown in Figure 8, which enlarges the small current density region, in the region of approximately $0-$ $0.01 \mathrm{~A} / \mathrm{cm}^{2}$, the curve fitted by model $\mathrm{K}$ deviates from the other two fitting curves expressively.

As seen in Table 6, the value of $\mathbf{E}_{\mathbf{O}}$ derived with model $\mathrm{K}$ is unreasonable and much smaller than the other two values, whereas model $\mathrm{F}$ and model $\mathrm{N}$ predict almost the same value 
TABLE 6: Values of fitting parameters obtained from three models (road test).

\begin{tabular}{lccc}
\hline & Model K & Model F & Model N \\
\hline$E_{\text {rev }}(\mathrm{V})$ & N/A & 1.2020 & N/A \\
$E_{\mathrm{OCV}}(\mathrm{V})$ & $0.7510\left(\mathbf{E}_{\mathrm{O}}\right)$ & 0.9574 & 0.9576 \\
$\mathbf{b}(\mathrm{V} /$ decade $)$ & $\left(0.9431\right.$ at $\left.1 \mathrm{~mA} \mathrm{~cm}^{-2}\right)$ & & \\
$\mathbf{R}\left(\Omega \mathrm{cm}^{2}\right)$ & 0.06412 & 0.06532 & 0.06516 \\
$\mathbf{i}_{\text {loss }}\left(\mathrm{A} \mathrm{cm}^{2}\right)$ & 0.3310 & 0.3287 & 0.3292 \\
$\mathbf{i}_{\mathbf{o}}\left(10^{-7} \mathrm{~A} \mathrm{~cm}^{2}\right)$ & N/A & 0.0006616 & 0.0006508 \\
\hline
\end{tabular}

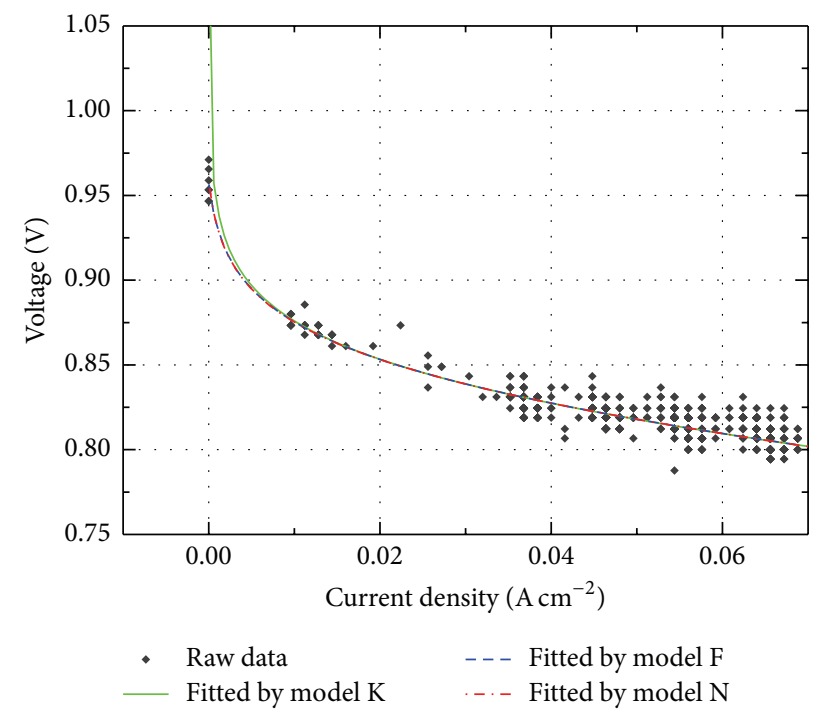

FIGURE 8: Comparison of measured and fitted polarization curves (small current densities) (road test).

of OCV. The fitted values of $\mathbf{b}$ and $\mathbf{R}$ derived with model $\mathrm{N}$ present extremely slight differences from those of model $\mathrm{F}$ but relatively obvious deviations from those of model K. The fitted internal current densities obtained from model $\mathrm{F}$ and model $\mathrm{N}$ exhibit only a slight distinction.

Through the foregoing analysis, it can be concluded that model $\mathrm{F}$ and model $\mathrm{N}$ show much better fitting characteristics than model $\mathrm{K}$ because of the minimum prediction error at OCV and small current density region. Although model N shows almost the same fitting characteristics as model $\mathrm{F}$, the most competitive advantage of model $\mathrm{N}$ is that the reactant pressures and operational temperature of the fuel cell stack are not indispensable for fitting. In addition, only three fitting parameters, one less than those of model $\mathrm{F}$, are required for model N. Each fitting parameter in model $\mathrm{N}$ has physical meaning, whereas $\mathbf{E}_{\mathbf{O}}$ in model $\mathrm{K}$ is merely a voltage fitting term. These features of model $\mathrm{N}$ are beneficial for researchers to extract polarization curves of fuel cells under a road test accurately and efficiently.

4.3. Advantages of the New Model. Among all proposed models, the model in this paper presents good fitting accuracy and the best advantage of convenience collectively. As shown in Table 1, the shortcomings of the existing models can be summarized as follows:

(1) Inability to fit open circuit voltage.

(2) Low fitting accuracy of small current densities.

(3) Requiring estimate of reversible voltage $E_{\text {rev }}$ or low fitting accuracy of $E_{\text {rev }}$.

(4) Requiring measured parameters, such as temperature and gas partial pressure.

(5) Requiring estimation of the smallest current density that causes the voltage to deviate from linearity $i_{d}$.

(6) Assuming that mass transfer loss occurs only in the high current density region.

(7) Requiring other empirical constants.

(8) Requiring measured limited current density $i_{\lim }$ and internal current density $i_{\text {loss }}$.

The model proposed in this work avoids all shortcomings and inconveniences mentioned above, and it is the author's belief that this is one of the most meaningful aspects of this work.

\section{Conclusion}

The purpose of this work is to obtain a simple, easy-to-use, efficient, and application-oriented polarization curve model with an acceptable fitting accuracy based on a review analysis. After summarizing numerous existing empirical fuel cell polarization curve models, a new model has been developed in this paper.

The following conclusions can be obtained:

(1) The new model contains five fitting parameters. Compared with most existing models, it does not require more fitting parameters.

(2) The new model has a compact formation, and its application only requires current-voltage data that are easy to measure. Hence, it is beneficial for applicationoriented investigations and industrial research.

(3) In the case of using bench test data, the new model provides almost ideal fitting characteristics with the entire range of current densities. Compared with other models, its advantages can be illustrated as follows: (a) the fitting curve definitely crosses the point of measured OCV; (b) the fitting curve presents excellent accuracy over the whole region of current densities. Moreover, all of the values of the fitting parameters derived from the new model appear in reasonable ranges. The fitted ohmic resistances of fuel cells ideally agree with the experimental data obtained through the current interruption test. As a consequence, the fitted results of the parameters can be references for research on fuel cell polarization curves. In addition, at present, under laboratory conditions, it is difficult to precisely measure some parameters, such as the limited current density and internal loss current density. The development of 
a model without any parameters that lack physical significance is the most important research direction of our future works.

(4) In the case of using road test data, it is difficult to precisely measure the steady pressures of reactants, operational temperature, limited current density, internal loss current density, and so on. As a consequence, the most prominent advantage of the new model is that excellent fitting characteristics over the whole current density region are presented without measuring any pressures or temperatures. Consequently, it is suitable for extracting the fuel cell stack performance accurately and efficiently from the discrete data sampled in a real road test.

\section{Competing Interests}

The authors declare that there are no competing interests regarding the publication of this paper.

\section{Acknowledgments}

The work was financially supported under the grants of the National Nature Science Foundation of China, Project no. 51275357.

\section{References}

[1] Y. Wang, K. S. Chen, J. Mishler, S. C. Cho, and X. C. Adroher, "A review of polymer electrolyte membrane fuel cells: technology, applications, and needs on fundamental research," Applied Energy, vol. 88, no. 4, pp. 981-1007, 2011.

[2] N. Duić, M. Lerer, and M. G. Carvalho, "Increasing the supply of renewable energy sources in island energy systems," International Journal of Sustainable Energy, vol. 23, no. 4, pp. 177-186, 2003.

[3] M. A. R. S. Al-Baghdadi, "A simple mathematical model of performance for proton exchange membrane fuel cells," International Journal of Sustainable Energy, vol. 26, no. 2, pp. 79-90, 2007.

[4] J. E. Choi and Y. C. Bae, "Swelling effect of a polymer electrolyte membrane on the development of a semi-empirical cell voltage model," Journal of Applied Electrochemistry, vol. 39, no. 9, pp. 1419-1424, 2009.

[5] S. A. Grigoriev, A. A. Kalinnikov, P. Millet, V. I. Porembsky, and V. N. Fateev, "Mathematical modeling of high-pressure PEM water electrolysis," Journal of Applied Electrochemistry, vol. 40, no. 5, pp. 921-932, 2010.

[6] A. A. Kulikovsky, "A simple and accurate fitting equation for half of the faradaic impedance arc of a PEM fuel cell," Journal of Electroanalytical Chemistry, vol. 738, pp. 108-112, 2015.

[7] A. A. Tahmasbi, A. Hoseini, and R. Roshandel, "A new approach to multi-objective optimisation method in PEM fuel cell," International Journal of Sustainable Energy, vol. 34, no. 5, pp. 283-297, 2015.

[8] J. Kim, S.-M. Lee, S. Srinivasan, and C. E. Chamberlin, "Modeling of proton exchange membrane fuel cell performance with an empirical equation," Journal of the Electrochemical Society, vol. 142, no. 8, pp. 2670-2674, 1995.
[9] J. H. Lee, T. R. Lalk, and A. J. Appleby, "Modeling electrochemical performance in large scale proton exchange membrane fuel cell stacks," Journal of Power Sources, vol. 70, no. 2, pp. 258-268, 1998.

[10] G. Squadrito, G. Maggio, E. Passalacqua, F. Lufrano, and A. Patti, "An empirical equation for polymer electrolyte fuel cell (PEFC) behaviour," Journal of Applied Electrochemistry, vol. 29, no. 12, pp. 1449-1455, 1999.

[11] D. Chu, R. Jiang, and C. Walker, "Analysis of PEM fuel cell stacks using an empirical current-voltage equation," Journal of Applied Electrochemistry, vol. 30, no. 3, pp. 365-370, 2000.

[12] Z. T. Xia and S. H. Chan, "Analysis of carbon-filled gas diffusion layer for $\mathrm{H}_{2}$ /air polymer electrolyte fuel cells with an improved empirical voltage-current model," International Journal of Hydrogen Energy, vol. 32, no. 7, pp. 878-885, 2007.

[13] L. Pisani, G. Murgia, M. Valentini, and B. D. Aguanno, "A new semi-empirical approach to performance curves of polymer electrolyte fuel cells," Journal of Power Sources, vol. 108, no. 1, pp. 192-203, 2002.

[14] S. D. Fraser and V. Hacker, "An empirical fuel cell polarization curve fitting equation for small current densities and no-load operation," Journal of Applied Electrochemistry, vol. 38, no. 4, pp. 451-456, 2008.

[15] H. Weydahl, M. S. Thomassen, B. T. Børresen, and S. MøllerHolst, "Response of a proton exchange membrane fuel cell to a sinusoidal current load," Journal of Applied Electrochemistry, vol. 40, no. 4, pp. 809-819, 2010.

[16] C. K. Poh, Z. Tian, N. Bussayajarn et al., "Performance enhancement of air-breathing proton exchange membrane fuel cell through utilization of an effective self-humidifying platinumcarbon catalyst," Journal of Power Sources, vol. 195, no. 24, pp. 8044-8051, 2010.

[17] S. Haji, "Analytical modeling of PEM fuel cell i-V curve," Renewable Energy, vol. 36, no. 2, pp. 451-458, 2011.

[18] M. V. Williams, H. R. Kunz, and J. M. Fenton, "Analysis of polarization curves to evaluate polarization sources in hydrogen/air PEM fuel cells," Journal of the Electrochemical Society, vol. 152, no. 3, pp. A635-A644, 2005.

[19] F. Barbir, PEM Fuel Cells: Theory and Practice, Elsevier, Amsterdam, Netherlands, 2005.

[20] Greenlight Innovation Corporation (GIC), G500 Fuel Cell Test Station, 2014, http://www.greenlightinnovation.com/database/ files/library/G500_Fuel_Cell_Test_Station.pdf.

[21] IEC (International Electrotechnical Commission), Fuel Cell Technologies-Part 7-1 Single Cell Test Methods for Polymer Electrolyte Fuel Cell (PEFC), IEC, 2010. 


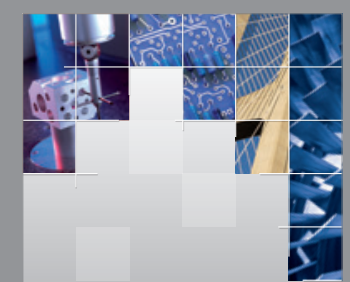

\section{Enfincering}
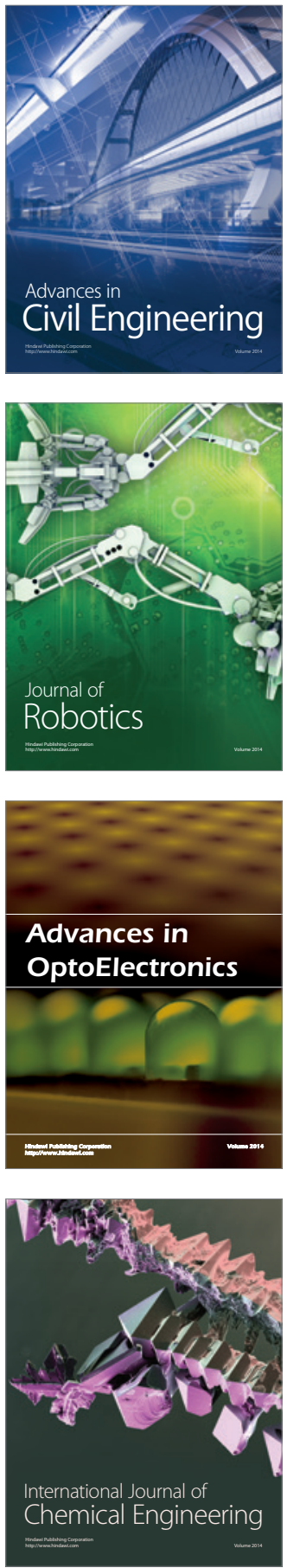

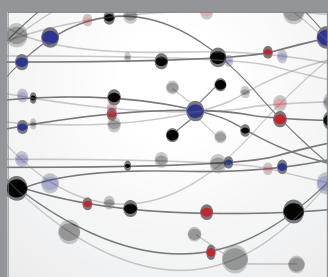

The Scientific World Journal

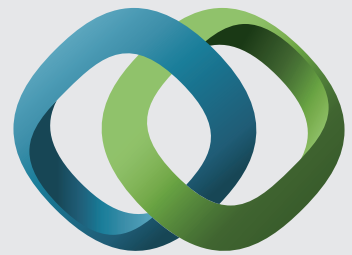

\section{Hindawi}

Submit your manuscripts at

http://www.hindawi.com
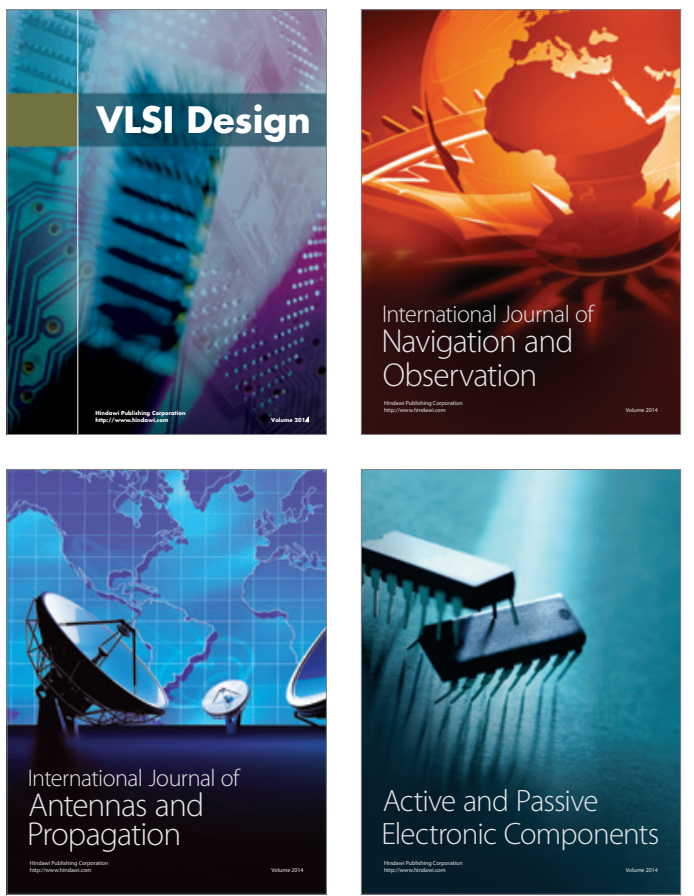
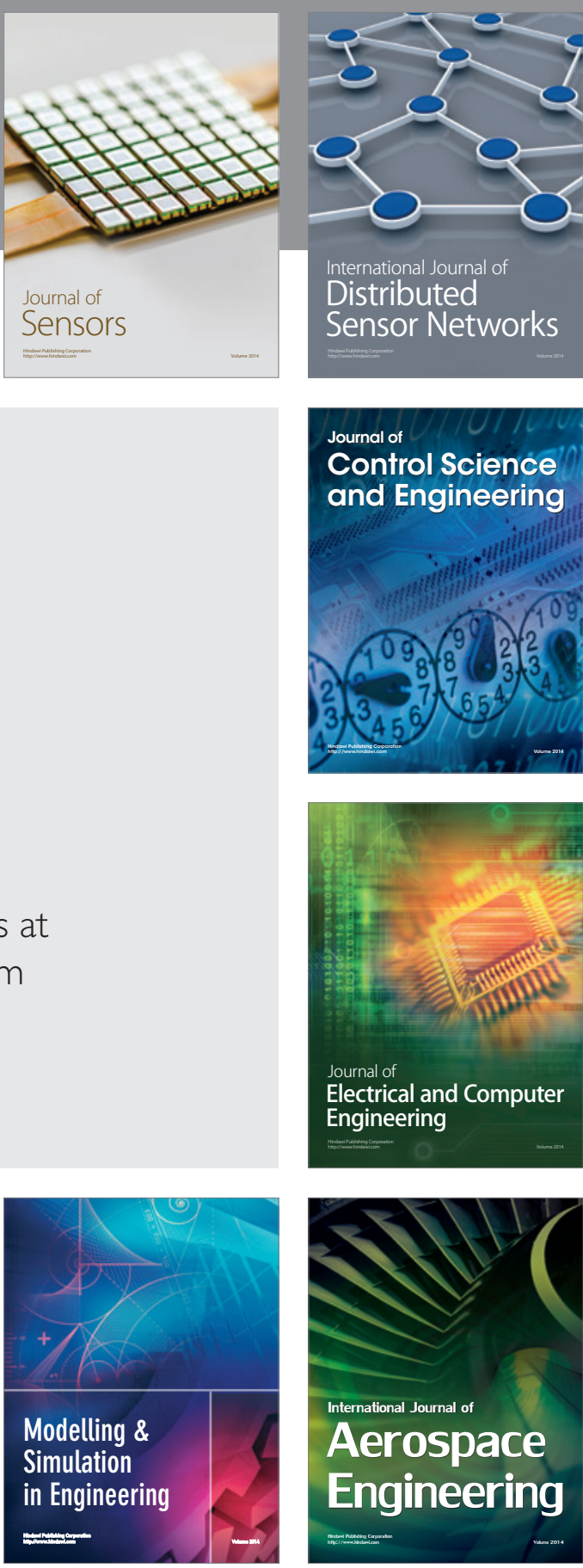

International Journal of

Distributed

Sensor Networks

Journal of

Control Science

and Engineering
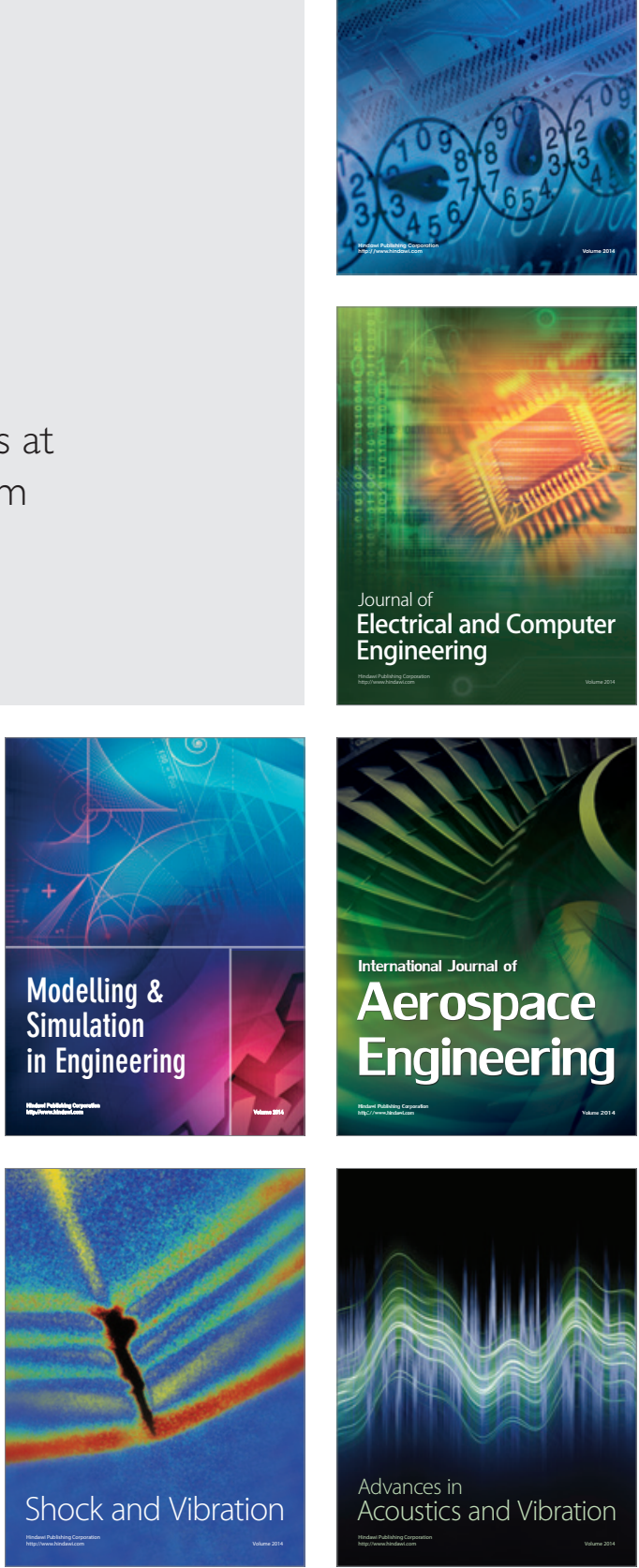